\title{
Comorbidades entre dependência química, distimia, HIV e HCV: Relato de caso
}

Comorbidity between addiction, dysthymia, HIV and HCV: Case report

\author{
Vanessa Fabiane Machado Gomes Marsden \\ Médica psiquiatra do Centro de Respostas Integradas (CRI) de Vila Real, Instituto da Droga e Toxicodependência (IDT), Portugal.
}

Recebido: 4/8/2008 - Aceito: 27/10/2008

\begin{abstract}
Resumo
Introdução: A comorbidade entre dependência química e doenças infectocontagiosas é bem conhecida, assim como a relação entre transtornos de humor e uso de substâncias. Entretanto, o transtorno distímico nestes pacientes recebe pouca atenção. Em parte, isso se justifica porque a realização do diagnóstico de distimia é mais difícil do que de outros transtornos do humor em razão do tempo de abstinência (2 anos) necessário para o diagnóstico, tendo em vista que toxicodependentes apresentam diversas recaídas durante o curso de suas vidas. As infecções pelos vírus HIV e HCV, frequentemente associadas ao consumo injetável de substâncias, contribuem para alterações do estado mental e o próprio tratamento pode causar diversas flutuações no humor. Relato de caso: O paciente é um homem de 40 anos de idade que apresenta comorbidade entre dependência química (heroína e álcool) e distimia, complicada por recaídas, consumo injetável e status sorológico positivo aos vírus HIV-1 e HCV. Conclusão: Pacientes dependentes químicos com comorbidades psiquiátricas e infectocontagiosas são desafiadores no que tange diagnóstico, tratamento e definição de abordagens terapêuticas para os diferentes problemas apresentados. Investigar e abordar adequadamente, entretanto, traz diversos benefícios na qualidade de vida do indivíduo afetado, assim como potenciais benefícios financeiros.
\end{abstract}

Marsden VFMG / Rev Psiq Clín. 2009;36(1):31-33

Palavras-chave: Comorbidade, transtorno distímico, dependência de heroína, alcoolismo.

\begin{abstract}
Background: Comorbidity between chemical dependence and infectious diseases is well known, as is the relationship between mood disorders and substance misuse. Nevertheless, dysthymia in these patients is not well explored. That is partly justified since the diagnoses of dysthymia is more difficult than other mood disorders due to the abstinence time (2 years) required for the diagnoses, and since addiction patients present several relapses during the course of their lifetimes. HIV and HCV infections, frequently associated to intravenous (IV) drug use, contribute to mental status deterioration and the treatment for such conditions can cause fluctuations on mood. Case report: A 40 years old patient with comorbidity between chemical dependence (heroin and alcohol) and dysthymia, complicated by relapses, IV drug use and serological status positive to HIV-1 and HCV. Conclusion: Addiction patients with psychiatric and infectious comorbidity are challenging to diagnose, treat and define therapeutical approaches to the different conditions. Proper clinical investigation and approach leads to benefits in health quality and potential financial benefits.
\end{abstract}

Marsden VFMG / Rev Psiq Clín. 2009;36(1):31-33

Key-words: Comorbidity, dysthymic disorder, heroin dependence, alcoholism. 


\section{Introdução}

A comorbidade entre toxicodependência e transtornos do humor foi vastamente descrita na literatura científica. Entretanto, a comorbidade entre dependência química, distimia e complicadores recebe pouca atenção ${ }^{1}$. Westermeyer et al. investigaram transtornos de substâncias e distimia em 1998 e deliberaram que a dificuldade diagnóstica provavelmente ocorre pelo fato de episódios depressivos e maníacos envolverem sintomas mais reconhecíveis do que a distimia ${ }^{2,3}$. Além disso, segundo esses autores, há dificuldade em diagnosticar distimia em toxicodependentes, pois os critérios diagnósticos para mania e episódio depressivo requerem estado de abstinência por $1 \mathrm{a} 2$ semanas, respectivamente, enquanto os sintomas para distimia devem estar presentes por pelo menos 2 anos $^{3}$.

Por outro lado, a comorbidade entre toxicodependência, doenças infectocontagiosas, tratamento e alterações cognitivas é largamente estudada e muito se tem produzido nesta área. As infecções pelos vírus da imunodeficiência humana (HIV) e pelos vírus da hepatite $\mathrm{C}(\mathrm{HCV})$, frequentemente associadas ao consumo injetável de substâncias, contribuem para alterações do estado mental e o próprio tratamento pode causar diversas flutuações no humor.

O caso relatado aqui é de um paciente que apresenta comorbidade entre dependência química de heroína e álcool e distimia, complicada por status sorológico positivo aos vírus HIV-1 e HCV.

\section{Relato de caso}

O paciente é um homem português de 40 anos de idade, que iniciou consumos de cannabis e álcool aos 22 anos, heroína aos 23, e consumos injetáveis, com partilha de material, aos 25. Com a evolução do quadro, o paciente passou a consumir apenas heroína, diariamente. Em 1997, iniciou terapêutica de manutenção com L-a-acetilmethadol (LAAM) em ambulatório público para tratamento da toxicodependência, onde ainda permanece, e foi diagnosticado como portador do vírus $\mathrm{HCV}$. Até então, teve diversas consultas psiquiátricas apenas para o tratamento da toxicodependência, sem outro diagnóstico. Entre 2001 e 2004, o paciente permaneceu abstinente por 2 anos e 4 meses, o que foi comprovado por análises de urina. Durante acompanhamento psiquiátrico em consequência de alterações do humor (insônia, falta de energia, apatia, baixa auto-estima, desesperança e dificuldades para tomar decisões), diagnosticou-se distimia. O tratamento da toxicodependência foi trocado para cloridrato de metadona em razão dos riscos de tratamento com LAAM, que foi abolido em todo Portugal. O paciente manteve quadro de humor estável com o uso de trazodona, paroxetina, lorazepam e oxazepam. Em 2004 , iniciou tratamento para hepatite $\mathrm{C}$ com interferon e foi diagnosticado como portador do vírus HIV. Tanto o tratamento com interferon quanto o conhecimento da infecção pelo HIV levaram à piora do quadro mental e o paciente apresentou recaída em opioides e álcool. Nessa nova fase, o paciente bebia mais de 30 unidades de álcool por dia, recaiu em consumos injetáveis de heroína e metadona (com partilha de material) e apresentava humor deprimido e afeto hipomodulante. Ele foi medicado com venlafaxina XR $150 \mathrm{mg} /$ dia e metadona $85 \mathrm{mg} /$ dia. Infelizmente, o paciente abusava dos medicamentos, ingerindo diversos comprimidos ao mesmo tempo, junto com bebidas alcoólicas. Como não houve melhora do quadro com a medicação e em virtude da desorganização apresentada pelo paciente, optou-se por nova proposta terapêutica com clomipramina $75 \mathrm{mg} / \mathrm{dia}$, olanzapina $10 \mathrm{mg} / \mathrm{dia}$, alprazolam $3 \mathrm{mg} /$ dia e metadona $105 \mathrm{mg} /$ dia e iniciou-se uma proposta integralista com apoio de psicoterapia, consultas de enfermagem, psiquiátricas e de serviço social: os comprimidos eram tomados pela manhã, com a dose de metadona, em frente a um profissional da enfermagem, que a seguir fornecia os comprimidos da noite para o paciente levar para casa. A abordagem em psicologia foi reestruturada para consultas mais breves, de cunho cognitivo e comportamental, e o paciente teve de aceitar um novo esquema de consultas com o serviço social. Este último teve como base o fato de que o paciente comparecia diariamente à instituição, solicitando consultas sociais, com o objetivo de chamar atenção para sua solidão e problemas financeiros. O paciente manteve-se abstinente de heroína e álcool (comprovado por exames de urina) com essa nova proposta terapêutica, apresentando melhora do humor, mas sem melhora das condições sociais.

\section{Comentários}

A dificuldade em estabelecer um diagnóstico de distimia em pacientes toxicodependentes dá-se ao fato de que é necessário um longo período de abstinência ( 2 anos) quando comparado a outros transtornos de humor. Entretanto, diagnosticar esses indivíduos é importante, pois altera os resultados esperados no tratamento e na qualidade de vida do paciente. Segundo a literatura, pacientes com comorbidade entre toxicodependência e distimia parecem receber mais tratamentos psiquiátricos não relacionados ao uso de substâncias do que os apenas toxicodependentes ${ }^{3}$. Pacientes toxicodependentes com distimia apresentam prognóstico e características que os diferenciam dos demais pacientes: toxicodependentes distímicos apresentam maior propensão a procurar tratamentos para abuso de substâncias, a serem admitidos em clínicas com esse fim mais frequentemente e para apresentar mais dias totais em tratamento, o que lembra o fenômeno "porta giratória" ilustra a procura incessante de atendimento, tipicamente apresentada por esses pacientes. Tal fato indica que diagnosticar tais indivíduos pode ser um bom investimento, tanto humanitário quanto financeiro. Apesar 
das dificuldades, investir em tratamento adequado da distimia em utilizadores de drogas resulta em melhor prognóstico médico e social e melhor qualidade de vida. Alguns estudos evidenciaram forte correlação entre sintomas depressivos e maior morbidade em pacientes com transtornos relacionados a substâncias quando comparados a pacientes apenas com transtornos relacionados à substâncias ${ }^{46}$. A identificação de transtornos de humor em pacientes em tratamento para abuso de dependências é reconhecidamente uma etapa importante do tratamento, pois quadros de humor depressivo podem levar à falha terapêutica, muitas vezes por incapacidade do paciente em cooperar com as medidas necessárias para estabelecer a abstinência.

Ao se avaliar comorbidades em dependência química, faz-se necessário analisar também as comorbidades infectocontagiosas comuns a essa população. No caso relatado neste artigo, o paciente apresentava sorologia positiva ao HIV e ao HCV. Na literatura, encontram-se diversas referências ao impacto que o diagnóstico de infecção pelo HIV causa no estado mental, desde transtornos de ajustamento a alterações cognitivas ${ }^{7-8}$. Também é reconhecido o impacto do tratamento para hepatite $\mathrm{C}$ no humor dos pacientes, como no caso citado, no qual interferon foi a droga escolhida, que tipicamente causa humor disfórico ou depressivo ${ }^{9}$. Recentemente, o papel que o próprio vírus $\mathrm{C}$ pode ter no humor e na qualidade de vida de pacientes não cirróticos está a ser estudado. Encontram-se na literatura artigos que indicam anormalidades metabólicas sugestivas de disfunção frontal-subcortical em pacientes com hepatite $\mathrm{C}$ não cirrótica ${ }^{10}$. A importância de se atentar para essa disfunção cognitiva reside no fato de que comumente leva à atenção e à concentração pobres e a dificuldades na memória recente que podem interferir na capacidade de aprender novas informações e piorar a qualidade de vida ${ }^{11}$.
Em conclusão, pacientes dependentes químicos com comorbidades psiquiátricas e infectocontagiosas são desafiadores no que tange a diagnóstico, tratamento e definição de abordagens terapêuticas para os diferentes problemas apresentados. Investigar e abordar adequadamente, entretanto, traz diversos benefícios na qualidade de vida do indivíduo afetado, assim como potenciais benefícios financeiros.

\section{Referências}

1. Kell MH. Opiate dependence, comorbidity and seasonality of birth. J Addict Disord. 1995;14:19-34.

2. Powell BJ, Penick EC, Nickel EJ, Liskow BI, Riesenmy KD, Campion SL, et al. Outcomes of co-morbid alcohol men: a 1-years follow-up. Alcohol Clin Exp Res. 1992;16:131-8.

3. Westermeyer J, Eames SL, Nugent S. Comorbid dysthymia and substance disorder: treatment history and cost. Am J Psychiatry. 1998;155:1556-60

4. Ravndal E, Vaglum P. Self-reported depression as a predictor of dropout in a hierarchical therapeutic community. J Subst Abuse Treat. 1994;11:471-9

5. Haywood TW, Kravitz HM, Grossman LS, Cavanaugh JL Jr, Davis JM, Lewis DA. Predicting the "revolving door" phenomenon among patients with schizophrenic, schizoaffective, and affective disorders. Am J Psychiatry. 1995;152:856-61.

6. Gogek EB. The dry drunk syndrome: subtype of depression? (letter). Am J Psychiatry. 1994;151:947-8.

7. Baum MK, Jayaweera DT, Duan R, Sales S, Lai S, Rafie C, et al. Quality of life, symptomatology and healthcare utilization in HIV/HCV co-infected drug users in Miami. J Addict Dis. 2008;27(2):37-48.

8. Whetten K, Reif S, Whetten R, Murphy-McMillan LK. Trauma, mental health, distrust, and stigma among HIV-positive persons: implications for effective care. Psychosom Med. 2008;Jun;70(5):531-8.

9. Malek-Ahmadi P, Hilsabeck RC. Neuropsychiatric complications of interferons: classification, neurochemical bases, and management. Ann Clin Psychiatry. 2007, Apr-Jun; 19 (2):113-123.

10. Forton DM, Allsop JM, Main J, Foster GR, Thomas HC, Taylor-Robinson SD. Evidence for a cerebral effect of the hepatitis $C$ virus. (letter). Lancet. 2000;358: 38-9.

11. Hilsabeck RC, Hassanein TI, Carlson MD, Ziegler EA, Perry W. Cognitive functioning and psychiatric symptomatology in patients with chronic hepatitis C. J Int Neuropsychol Soc. 2003;9(6):847-54. 